\title{
Digit-recurrence algorithm for computing Euclidean norm of a $3-D$ vector
}

\author{
Naofumi Takagi and Seiji Kuwahara \\ Department of Information Engineering \\ Nagoya University \\ Nagoya 464-8603, Japan \\ email: ntakagi@nuie.nagoya-u.ac.jp
}

\begin{abstract}
A digit-recurrence algorithm for computing the Euclidean norm of a 3-dimensional vector is proposed. Starting from the vector component with the highest order of magnitude as the initial value of partial result, correcting-digits produced by the recurrence are added to it step by step. Partial products of the squares of the other two components are added to the residual, step by step. The addition/subtractions in the recurrence are performed without carry/borrow propagation by the use of a redundant representation of the residual. An extension of the on-the-fly conversion algorithm is used for updating the partial result. Different specific versions of the algorithm are possible, depending on the radix, the redundancy factor of the correcting-digit set, the type of representation of the residual, and the correcting-digit selection function.
\end{abstract}

\section{Introduction}

Computation of the Euclidean norm of a 3dimensional (3-D) vector often appears in 3-D Computer Graphics for, e.g., normalizing the vector. Note that not its square but the Euclidean norm itself is required for normalizing a vector. Usually, the Euclidean norm is computed through three squarings, two additions and one square rooting. We take these operations together into one digit-recurrence, and propose a digitrecurrence algorithm.

In the algorithm to be proposed, starting with the vector component with the highest order of magnitude among the three as the initial value of partial result, we add correcting-digits produced by the recurrence to it step by step for obtaining the norm. We add partial products of the squares of the other two components to the residual, step by step. We select each correcting-digit from a redundant digit set by estimating the residual and the partial result. We perform addition/subtractions appearing in the recurrence without carry/borrow propagation by representing the residual in a redundant representation such as carrysave form or signed-digit representation [1]. We extend the on-the-fly conversion algorithm [2] for updating the partial result.

Different specific versions of the algorithm are possible, depending on the radix, the redundancy factor of the correcting-digit set, the type of representation of the residual, and the correcting-digit selection function, as digit-recurrence algorithms for division or square rooting [3].

Any version of the algorithm can be implemented as a sequential circuit or a combinational circuit. Pipelining can also be used. The implementations have a regular array structure suitable for VLSI. They are efficient both in time and area.

In this paper, we first define the computation of the Euclidean norm of a 3-D vector to be considered. Then, we show a general algorithm in Section 3. We show a radix-2 version of the algorithm in Section 4, and consider its implementations in Section 5.

\section{Computation of the Euclidean norm of a 3 -D vector}

We consider the computation of the mantissa part of the Euclidean norm of a $3-D$ vector represented as a triple of floating-point numbers, $\left(F_{X}, F_{Y}, F_{Z}\right)$, where $F_{X}=(-1)^{S_{X}} \cdot 2^{E_{X}} \cdot M_{X}, F_{Y}=(-1)^{S_{Y}} \cdot 2^{E_{Y}} \cdot M_{Y}$, and $F_{Z}=(-1)^{S_{Z}} \cdot 2^{E_{Z}} \cdot M_{Z}$. Here, $S_{X}(\in\{0,1\}), E_{X}$, and $M_{X}\left(\frac{1}{2} \leq M_{X}<1\right)$ are the sign bit, the exponent part, and the mantissa part of $F_{X}$, respectively.

Without loss of generality, we assume that $F_{X}$ has 
the highest order of magnitude among the three components. Namely, $E_{X} \geq E_{Y}$ and $E_{X} \geq E_{Z}$. Then, the Euclidean norm of the vector is $\sqrt{F_{X}^{2}+F_{Y}^{2}+F_{Z}^{2}}=$ $\sqrt{X^{2}+Y^{2}+Z^{2}} \cdot 2^{E_{X}}$, where $X=M_{X}, Y=M_{Y}$. $2^{E_{Y}-E_{X}}$, and $Z=M_{Z} \cdot 2^{E_{Z}-E_{X}}$. Therefore, in this paper, we consider the computation of $P=$ $\sqrt{X^{2}+Y^{2}+Z^{2}}$, where $\frac{1}{2} \leq X<1,0 \leq Y<1$, and $0 \leq Z<1 . \frac{1}{2} \leq P<\sqrt{3}$ holds.

We assume that $X, Y$, and $Z$ are represented as $n$-digit, $(n+h)$-digit, and $(n+h)$-digit $r$-ary fractions where $r=2^{b}$, respectively. Namely, $X, Y$ and $Z$ are represented as $\left[. x_{1} x_{2} \cdots x_{n}\right],\left[. y_{1} y_{2} \cdots y_{n+h}\right]$, and $\left[. z_{1} z_{2} \cdots z_{n+h}\right]$, respectively, and $X=\sum_{i=1}^{n} x_{i} r^{-i}$, $Y=\sum_{i=1}^{n+h} y_{i} r^{-i}$, and $Z=\sum_{i=1}^{n+h} z_{i} r^{-i} . h$ is a nonnegative integer determined for each version of the proposed algorithm. We will discuss on $h$ in subsection 3.2 .

We intend to obtain $P$ so that it satisfies $\left|\sqrt{X^{2}+Y^{2}+Z^{2}}-P\right|<r^{-n}$. We assume that $P$ is represented as an $(n+1)$-digit $r$-ary number, $\left[p_{0} \cdot p_{1} p_{2} \cdots p_{n}\right]$.

\section{General algorithm}

\subsection{Recurrence}

Here, we derive a general digit-recurrence for radix $r$. We assume the radix is a power of 2, i.e., $r=2^{b}$.

Different from digit-recurrence algorithms for division or square rooting [3], the result is obtained by adding correcting-digit $q_{j}$ produced by the recurrence to the initial value $P[0]$ of the partial result. We will discuss on the value of $P[0]$ later. Furthermore, partial products of $Y^{2}$ and $Z^{2}$, i.e., $Y \cdot y_{j}$ and $Z \cdot z_{j}$ are added to the residual, step by step.

Let $P[j]$ be the partial result after $j$ iterations. Then, $P[j]=P[0]+\sum_{i=1}^{j} q_{i} r^{-i}$. The recurrence on the partial result is

$$
P[j+1]:=P[j]+q_{j+1} r^{-j-1} .
$$

We select the correcting-digit $q_{j+1}$ from a redundant digit set $\{-a, \cdots,-1,0,1, \cdots, a\}$, where $\frac{r}{2} \leq a<r$. The final result is $P=P[n]=P[0]+\sum_{i=1}^{n} q_{i} r^{-i}$. The result has to be computed for $n$-digit precision. Namely,

$$
\left|\sqrt{X^{2}+Y^{2}+Z^{2}}-P\right|<r^{-n}
$$

We define a residual (or scaled partial remainder) $W[j]$ as

$$
W[j]=r^{j}\left(X^{2}+Y \cdot Y_{j+h}+Z \cdot Z_{j+h}-P[j]^{2}\right),
$$

where $Y_{j+h}=\left[. y_{1} y_{2} \cdots y_{j+h}\right]$ and $Z_{j+h}=$ $\left[z_{1} z_{2} \cdots z_{j+h}\right]$. We will discuss on $h$ in the next subsection. Subtracting $r$ times (3) from the formula for $W[j+1]$, we get the recurrence on the residual:

$$
\begin{array}{r}
W[j+1]:=r W[j]+Y y_{j+h+1} r^{-h}+Z z_{j+h+1} r^{-h} \\
-2 P[j] q_{j+1}-q_{j+1}^{2} r^{-j-1} .
\end{array}
$$

We will discuss on selection of the correcting-digit $q_{j+1}$ in the next subsection.

From $(2),\left(P-r^{-n}\right)^{2}<X^{2}+Y^{2}+Z^{2}<\left(P+r^{-n}\right)^{2}$ must hold. Since $P=P[j]+\sum_{i=j+1}^{n} q_{i} r^{-i}, Y=Y_{j+h}+$ $\sum_{i=j+h+1}^{n+h} y_{i} r^{-i}$ and $Z=Z_{j+h}+\sum_{i=j+h+1}^{n+h} z_{i} r^{-i}$, from (3),

$2 P[j]\left(\sum_{i=j+1}^{n} q_{i} r^{-i}-r^{-n}\right)+\left(\sum_{i=j+1}^{n} q_{i} r^{-i}-r^{-n}\right)^{2}$ $<r^{-j} W[j]+Y \cdot \sum_{i=j+h+1}^{n+h} y_{i} r^{-i}+Z \cdot \sum_{i=j+h+1}^{n+h} z_{i} r^{-i}$ $<2 P[j]\left(\sum_{i=j+1}^{n} q_{i} r^{-i}+r^{-n}\right)+\left(\sum_{i=j+1}^{n} q_{i} r^{-i}+r^{-n}\right)^{2}$ must hold. Since the minimum and the maximum correcting-digit values are $-a$ and $a$, respectively, $-2 P[j] \rho r^{-j}+\rho^{2} r^{-2 j}$

$<r^{-j} W[j]+Y \cdot \sum_{i=j+h+1}^{n+h} y_{i} r^{-i}+Z \cdot \sum_{i=j+h+1}^{n+h} z_{i} r^{-i}$ $<2 P[j] \rho r^{-j}+\rho^{2} r^{-2 j}$

where $\rho=a /(r-1)$ is the redundancy factor of the correcting-digit set. Since $0 \leq Y \cdot \sum_{i=j+h+1}^{n+h} y_{i} r^{-i}+Z$. $\sum_{\text {as }}^{n+h} z_{i=j+h+1} z_{i} r^{-i}<2 r^{-j-h}$, we get the bounds for $W[j]$

$$
-2 P[j] \rho+\rho^{2} r^{-j}<W[j]<2 P[j] \rho+\rho^{2} r^{-j}-2 r^{-h} .
$$

Now, we consider the initial values of the residual and the partial result. Initially,

$-2 P[0] \rho+\rho^{2}$

$<W[0]=X^{2}+Y \cdot Y_{h}+Z \cdot Z_{h}-P[0]^{2}$

$<2 P[0] \rho+\rho^{2}-2 r^{-h}$

must hold. Since $\frac{1}{2}<\rho \leq 1$, we can satisfy these bounds by letting

$$
P[0]=X+2^{-1} .
$$

Then,

$$
W[0]=Y \cdot Y_{h}+Z \cdot Z_{h}-X-2^{-2} .
$$

The larger $h$ is, the more the required computations for obtaining $W[0]$.

The algorithm for computing the Euclidean norm consists in performing $n$ iterations of the recurrences (1) and (4). In each iteration, we first produce the shifted residual $r W[j]$, and the partial products $Y y_{j+h+1} r^{-h}$ and $Z z_{j+h+1} r^{-h}$. Then, we select the correcting-digit $q_{j+1}$ by examining the shifted residual and the partial result $P[j]$. Finally, we perform the addition/subtractions in the recurrence (4).

The general algorithm is summarized as follows: 


\section{Algorithm [3DEN]}

Step 1:

$$
\begin{aligned}
& P[0]:=X+2^{-1} \\
& W[0]:=Y \cdot Y_{h}+Z \cdot Z_{h}-X-2^{-2} ;
\end{aligned}
$$

Step 2:

$$
\begin{aligned}
& \text { for } j:=0 \text { to } n-1 \text { do begin } \\
& \qquad \begin{array}{r}
\text { Select } q_{j+1} \text { from }\{-a, \cdots,-1,0,1, \cdots, a\} ; \\
P[j+1]:=P[j]+q_{j+1} r^{-j-1} ; \\
W[j+1]:=r W[j]+Y y_{j+h+1} r^{-h}+Z z_{j+h+1} r^{-h} \\
\quad-2 P[j] q_{j+1}-q_{j+1}^{2} r^{-j-1} ;
\end{array}
\end{aligned}
$$

end

We will discuss on selection of the correcting-digit $q_{j+1}$ in the next subsection.

We can increase the speed of the implementation with a small increase in hardware complexity by performing the addition/subtractions in the recurrence on the residual without carry/borrow propagation by the use of a redundant representation. Therefore, in this paper, we concentrate on this type of implementations. Namely, we represent the residual $W[j]$ in a redundant representation, such as the carry-save form or the (binary) signed-digit representation [1], and perform the addition/subtractions without carry/borrow propagation. Since $-4<W[j]<4$, we can represent $W[j]$ by either a two's complement carry-save form with 3-bit integer part (including the sign bit) or a binary signeddigit representation with 3 -bit integer part.

Although we may represent the partial result $P[j]$ in a redundant representation as well, we keep the nonredundant representation of it by an extension of the on-the-fly conversion. We have to extend the on-the-fly conversion algorithm, because the initial value of the partial result is not 0 but $X+2^{-1}$. We will discuss on this in subsection 3.3 .

\subsection{Correcting-digit selection}

We have to select correcting digit $q_{j+1}$ from $\{-a, \cdots,-1,0,1, \cdots, a\}$ so that the bounds for $W[j+$ 1], i.e., $-2 P[j+1] \rho+\rho^{2} r^{-j-1}<W[j+1]<2 P[j+$ 1] $\rho+\rho^{2} r^{-j-1}-2 r^{-h}$, are satisfied. Recall the recurrence on the residual (4). $q_{j+1}$ depends on the shifted residual $r W[j]$, the partial products $Y y_{j+h+1} r^{-h}$ and $Z z_{j+h+1} r^{-h}$, and the partial result $P[j]$. We define $V[j]$ as

$$
V[j]=r W[j]+Y y_{j+h+1} r^{-h}+Z z_{j+h+1} r^{-h} .
$$

Let the interval of $V[j]$ where $q_{j+1}=k$ can be selected be $\left[L_{k}[j], U_{k}[j]\right)$. Then,

$$
L_{k}[j]=2 P[j](k-\rho)+(k-\rho)^{2} r^{-j-1},
$$

and

$$
U_{k}[j]=2 P[j](k+\rho)+(k+\rho)^{2} r^{-j-1}-2 r^{-h} .
$$

Note that the lower bound of the interval for $k=-a$ and the upper bound of the interval for $k=a$ are equal to the lower bound and the upper bound of $V[j]$, respectively.

The continuity condition $U_{k-1}[j] \geq L_{k}[j]$ yields

$$
(2 \rho-1)\left(2 P[j]+(2 k-1) r^{-j-1}\right)-2 r^{-h} \geq 0 .
$$

$h$ must be determined so that (11) is satisfied. The smaller $\rho$ is, the larger $h$ must be.

The overlap between consecutive selection intervals is given by $(2 \rho-1)\left(2 P[j]+(2 k-1) r^{-j-1}\right)-2 r^{-h}$. This overlap is used to simplify the selection function. The larger $h$ is, the larger the overlap is, and hence, the simpler the selection function may be.

As stated, $q_{j+1}$ depends on $V[j](=r W[j]+$ $\left.Y y_{j+h+1} r^{-h}+Z z_{j+h+1} r^{-h}\right)$ and $P[j]$. Using the overlap, we can select $q_{j+1}$ by estimates of them. We can reduce the effect of $Y y_{j+h+1} r^{-h}$ and $Z z_{j+h+1} r^{-h}$ by making $h$ larger. Let the correcting-digit selection function be $\operatorname{Select}(\hat{V}[j], \hat{P}[j])$ where $\hat{V}[j]$ and $\hat{P}[j]$ are estimates of $V[j]$ and $P[j]$, respectively. Then, the correction-digit selection function is described by the set of selection constants, $\left\{m_{k}(\hat{P}[j]) \mid k \in\{-a+\right.$ $1, \cdots,-1,0,1, \cdots, a\}\}$, where $q_{j+1}=k$ if $m_{k}(\hat{P}[j]) \leq$ $\hat{V}[j]<m_{k+1}(\hat{P}[j])$.

We determine $h$ so that the partial products have no effect on the correcting-digit selection. Note that $0 \leq Y y_{j+h+1} r^{-h}<(r-1) r^{-h}$, and $0 \leq Z z_{j+h+1} r^{-h}<$ $(r-1) r^{-h}$. We obtain $\hat{V}[j]$ by truncating $r W[j]$, which is in a redundant representation, to $t$ fractional bits. (Note that not $r$-ary digits but bits.) We obtain $\hat{P}[j]$ by truncating $P[j]$ to $d$ fractional bits. (Note again that not $r$-ary digits but bits.) Since $P[j]$ is in the non-redundant representation as to be shown in the next subsection, $\hat{P}[j] \leq P[j]<\hat{P}[j]+2^{-d}$.

When $W[j]$ is in the carry-save form, $\hat{V}[j] \leq$ $r W[j]<\hat{V}[j]+2^{-t+1}$. Therefore,

$$
m_{k}(\hat{P}[j]) \geq \max _{\hat{P}[j]}\left(L_{k}[j]\right),
$$

and

$\left(m_{k}(\hat{P}[j])-2^{-t}\right)+2^{-t+1}+2(r-1) r^{-h} \leq \min _{\hat{P}[j]}\left(U_{k-1}[j]\right)$

must be satisfied. $\max _{\hat{P}[j]}\left(L_{k}[j]\right)$ denotes the maximum value of the lower bound of the interval of $V[j]$ where $q_{j+1}=k$ can be selected when the estimate of $P[j]$ is $\hat{P}[j] . \min _{\hat{P}[j]}\left(U_{k-1}[j]\right)$ denotes the minimum 
value of the upper bound of the interval of $V[j]$ where $q_{j+1}=k-1$ can be selected when the estimate of $P[j]$ is $\hat{P}[j]$. Note that the maximum value of $\hat{V}[j]$ for which $k-1$ is selected as $q_{j+1}$ is $m_{k}(\hat{P}[j])-2^{-t}$.

When $W[j]$ is in the binary signed-digit representation, $\hat{V}[j]-2^{-t} \leq r W[j]<\hat{V}[j]+2^{-t}$, and hence,

$$
m_{k}(\hat{P}[j])-2^{-t} \geq \max _{\hat{P}[j]}\left(L_{k}[j]\right)
$$

and

$$
\left(m_{k}(\hat{P}[j])-2^{-t}\right)+2^{-t}+2(r-1) r^{-h} \leq \min _{\hat{P}[j]}\left(U_{k-1}[j]\right)
$$

must be satisfied.

In both cases, the minimum overlap required for a feasible correcting-digit selection is

$$
\min _{\hat{P}[j]}\left(U_{k-1}[j]\right)-\max _{\hat{P}[j]}\left(L_{k}[j]\right) \geq 2^{-t}+2(r-1) r^{-h} .
$$

From (9) and (10), for $k>0$

$$
\max _{\hat{P}[j]}\left(L_{k}[j]\right)=2\left(\hat{P}[j]+2^{-d}\right)(k-\rho)+(k-\rho)^{2} r^{-j-1},
$$

and

$$
\min _{\hat{P}[j]}\left(U_{k-1}[j]\right)=2 \hat{P}[j](k-1+\rho)+(k-1+\rho)^{2} r^{-j-1}-2 r^{-h} .
$$

For $k \leq 0$

$$
\max _{\hat{P}[j]}\left(L_{k}[j]\right)=2 \hat{P}[j](k-\rho)+(k-\rho)^{2} r^{-j-1},
$$

and

$$
\begin{array}{r}
\min _{\hat{P}[j]}\left(U_{k-1}[j]\right)=2\left(\hat{P}[j]+2^{-d}\right)(k-1+\rho) \\
+(k-1+\rho)^{2} r^{-j-1}-2 r^{-h} .
\end{array}
$$

If we use these values to determine the correcting-digit selection, since they depend on $j$, a different selection function might result for different $j$.

Here, we try to have a single selection function. We develop expressions that are independent of $j$, as follows. For $k>0, \min _{\hat{P}[j]}\left(U_{k-1}[j]\right)-\max _{\hat{P}[j]}\left(L_{k}[j]\right)$ $\geq\left(2 \hat{P}[j](k-1+\rho)-2 r^{-h}\right)-\left(2\left(\hat{P}[j]+2^{-d}\right)(k-\rho)+(k-\right.$ $\left.\bar{\rho})^{2} r^{-1}\right)=2 \hat{P}[j](2 \rho-1)-2^{-d+1}(k-\rho)-(k-\rho)^{2} r^{-1}-$ $2 r^{-h}$. For $k \leq 0, \min _{\hat{P}[j]}\left(U_{k-1}[j]\right)-\max _{\hat{P}[j]}\left(L_{k}[j]\right)$ $\geq\left(2\left(\hat{P}[j]+2^{-d}\right)(k-1+\rho)-2 r^{-h}\right)-(2 \hat{P}[j](k-\rho)+$ $\left.(k-\rho)^{2} r^{-1}\right)=2 \hat{P}[j](2 \rho-1)+2^{-d+1}(k-1+\rho)-$ $(k-\rho)^{2} r^{-1}-2 r^{-h}$. The worst case is $\hat{P}[j]=2^{-1}$ and $k=-a+1$.

When $r=2, a=1$ and $\rho=1$. The worst value is $2^{-1}-2^{-h+1}$. From (16), we can get a single selection function, if $2^{-1}-2^{-h+1} \geq 2^{-t}+2^{-h+1}$, i.e., $2^{-1}-$ $2^{-h+2} \geq 2^{-t}$, is satisfied. $t=2$ and $h=4$ is a possible solution. $d$ can be any integer.

When $r>2$, i.e, $r \geq 4$, the worst value is $(2 \rho-1)-$ $2^{-d+1} \rho(r-2)-(\rho r-\overline{1})^{2} r^{-1}-2 r^{-h}<(2 \rho-1)-(\rho r-$ $1)^{2} r^{-1}<-\rho^{2} r+4 \rho-1 \leq-\left(4 \rho^{2}-4 \rho+1\right)=-(2 \rho-1)^{2} \leq$ 0 . This means that no single selection function for all $j$ exists, as the case of square rooting [4]. Therefore, we try to find $J$ so that a single selection function can be used for $j \geq J$. Then, we consider the cases for $j<J$ separately. We can get a single selection function used for $j \geq J$, if $(2 \rho-1)-2^{-d+1} \rho(r-2)-(\rho r-1)^{2} r^{-J-1}-$ $2 r^{-h} \geq 2^{-t}+2(r-1) r^{-h}$, i.e., $2 \rho-1-(\rho r-1)^{2} r^{-J-1}-$ $2 r^{-h+1} \geq 2^{-t}+2^{-d+1} \rho(r-2)$, is satisfied. For specific values of $r$ and $\rho$, we can determine the values of $J, t$, $d$, and $h$. These are used to determine a single selection function for $j \geq J$. For example, for $r=4$ and $a=2$ ( $\rho=\frac{2}{3}$ ), a possible solution is $J=1, t=4, d=5$, and $h=6$. For $r=4$ and $a=3(\rho=1)$, a possible solution is $J=1, t=3, d=4$, and $h=4$.

\subsection{Representation of the partial result - Extension of the on-the-fly conversion}

Here, we consider representation of the partial result $P[j]$. We intend to have the non-redundant $r$-ary representation (with digit set $\{0,1, \cdots, r-1\}$ ) of $P[j]$ by an extension of the on-the-fly conversion.

Different from digit-recurrence algorithms for division and square rooting where the partial result after $j$ iterations is a $j$-digit number [3], $P[j]$ is an $n+1$-digit number. Since the $j+1$-th digit of (the non-redundant representation of) $P[j]$ is in the range $[0, r-1]$ and $q_{j+1}$ is selected from $\{-a, \cdots,-1,0,1, \cdots, a\}$, there are three possible carry values, i.e., $0,-1$ and 1 , into the $j$-th place of $P[j+1]$. Note that in digit-recurrence algorithms for division and square rooting, there are only two possible carry values, i.e., 0 and -1 .

Therefore, we extend the on-the-fly conversion algorithm [2]. We keep the non-redundant representations of $P[j]+r^{-j}$ and $P[j]-r^{-j}$ instead of those of $P[j]$ and $P[j]-r^{-j}$. Let the non-redundant representations of $P[j]+r^{-j}$ and $P[j]-r^{-j}$ be $P[j]^{+}$and $P[j]^{-}$, respectively. Note that for $i$ 's such that $i>j$, the $i$-th digit of $P[j]^{+}$and that of $P[j]^{-}$are identical and are equal to that of $X$, i.e., $x_{i}$. The non-redundant representation of $P[j]$ is obtained by either rewriting the $j$-th digit of $P[j]^{-}$to $p[j]_{j}^{-}+1$ or rewriting the $j$-th digit of $P[j]^{+}$ to $p[j]_{j}^{+}-1$, accordingly as $p[j]_{j}^{+}=0$ or $\neq 0$, where $p[j]_{j}^{-}$and $p[j]_{j}^{+}$denote the $j$-th digit of $P[j]^{-}$and that of $P[j]^{+}$, respectively. 
Table 1. The rule for the extended on-the-fly conversion

\begin{tabular}{|c|c|c|c|c|c|c|c|}
\hline & & \multicolumn{3}{|c|}{$P[j+1]^{+}$} & \multicolumn{3}{|c|}{$P[j+1]^{-}$} \\
\hline$g$ & $p[j]_{j}^{+}$ & $P[j]^{+} / P[j]^{-}$ & $p[j+1]_{j}^{+}$ & $p[j+1]_{j+1}^{+}$ & $P[j]^{+} / P[j]^{-}$ & $p[j+1]_{j}^{-}$ & $p[j+1]_{j+1}^{-}$ \\
\hline$\leq-2$ & & $P[j]^{-}$ & $\overline{p[j]_{j}^{-}}$ & $g+r+1$ & $P[j]^{-}$ & $\overline{p[j]_{j}^{-}}$ & $g+r-1$ \\
\hline \multirow[t]{2}{*}{$-1,0$} & $=0$ & $P[j]^{-}$ & $p[j]_{j}^{-}+1$ & $g+1$ & \multirow[t]{2}{*}{$P[j]^{-}$} & \multirow[t]{2}{*}{$p[j]_{j}^{-}$} & \multirow[t]{2}{*}{$g+r-1$} \\
\hline & $\neq 0$ & $P[j]^{+}$ & $p[j]_{j}^{+}-1$ & $g+1$ & & & \\
\hline \multirow[t]{2}{*}{$2, \cdots, r-2$} & $=0$ & $P[j]^{-}$ & $p[j]_{j}^{-}+1$ & $g+1$ & $P[j]^{-}$ & $p[j]_{j}^{-}+1$ & $g-1$ \\
\hline & $\neq 0$ & $P[j]^{+}$ & $p[j]_{j}^{+}-1$ & $g+1$ & $P[j]^{+}$ & $p[j]_{j}^{+}-1$ & $g-1$ \\
\hline \multirow[t]{2}{*}{$r-1, r$} & $=0$ & \multirow[t]{2}{*}{$P[j]^{+}$} & \multirow[t]{2}{*}{$p[j]_{j}^{+}$} & \multirow[t]{2}{*}{$g-r+1$} & $P[j]^{-}$ & $p[j]_{j}^{-}+1$ & $g-1$ \\
\hline & $\neq 0$ & & & & $P[j]^{+}$ & $p[j]_{j}^{+}-1$ & $g-1$ \\
\hline$\geq r+1$ & & $P[j]^{+}$ & $p[j]_{i}^{+}$ & $g-r+1$ & $P[j]^{+}$ & $p[j]_{i}^{+}$ & $g-r-1$ \\
\hline
\end{tabular}

Table 2. The rule for generating the adder input

\begin{tabular}{|c|c|c|c|c|}
\hline$g^{\prime}$ & $p[j]_{j}^{+}$ & $P[j]^{+} / P[j]$ & $j$-th digit & $j+1$-th and $j+2$-th digits \\
\hline$<0$ & & $P[j]^{-}$ & $\overline{p[j]_{j}^{-}}$ & $\overline{g^{\prime}+r^{2}}$ \\
\hline \multirow[t]{2}{*}{$0, \cdots, r^{2}-1$} & $=0$ & $P[j]^{-}$ & $p[j]_{j}^{-}+1$ & $g^{\prime}$ \\
\hline & $\neq 0$ & $P[j]^{+}$ & $p[j]_{j}^{+}-1$ & $g^{\prime}$ \\
\hline$\geq r^{2}$ & & $P[j]^{+}$ & $p[j]_{j}^{+}$ & $g^{\prime}-r^{2}$ \\
\hline
\end{tabular}

Initially,

$$
P[0]^{+}=\left[2 \cdot\left(x_{1}-\frac{r}{2}\right) x_{2} x_{3} \cdots x_{n}\right]
$$

and

$$
P[0]^{-}=\left[0 .\left(x_{1}-\frac{r}{2}\right) x_{2} x_{3} \cdots x_{n}\right]
$$

Note that $P[0]=X+2^{-1}=\left[1 .\left(x_{1}-\frac{r}{2}\right) x_{2} x_{3} \cdots x_{n}\right]$. Recall that since $X \geq \frac{1}{2}, x_{1} \geq \frac{r}{2}$. (When $r=2$, the integer part of $P[0]^{+}$is [10] in binary.)

We can obtain $P[j+1]^{+}$and $P[j+1]^{-}$by selecting $P[j]^{+}$or $P[j]^{-}$and rewriting the $j$-th and the $j+1$-th digits of the selected one, according to the rule shown in Table 1. $g$ denotes $x_{j+1}+q_{j+1}$. Note that when $r=2$, there are only two groups, i.e., $g$ is -1 or 0 and $1(=r-1)$ or $2(=r)$. The rule for $r=2$ is show in Table 3 in the next section.

Let us consider the case of $g=r$ as an example. The upper digits of $P[j+1]^{+}$down to the $j$-th place must be those of the non-redundant representation of $P[j]+r^{-j}$. Therefore, we select $P[j]^{+}$and rewrite its $j+1$-th digit to $1(=g-r+1)$. On the other hand, the upper digits of $P[j+1]^{-}$down to the $j$-th place must be those of the non-redundant representation of $P[j]$. Therefore, if $p[j]_{j}^{+}=0$, we select $P[j]^{-}$and rewrite its $j$-th and $j+1$-th digits to $p[j]_{j}^{-}+1$ and $r-1(=g-1)$ respectively, and otherwise, we select $P[j]^{+}$and rewrite its $j$-th and $j+1$-th digits to $p[j]_{j}^{+}-1$ and $r-1(=g-1)$ respectively.
Recall the recurrence on the residual (4). We can make this computation simpler by providing the nonredundant representation of $-2 P[j] q_{j+1}-q_{j+1}^{2} r^{-j-1}=$ $-2 q_{j+1}\left(P[j]+\frac{1}{2} q_{j+1} r^{-j-1}\right)$. We can obtain the nonredundant representation of $P[j]+\frac{1}{2} q_{j+1} r^{-j-1}$ by selecting $P[j]^{+}$or $P[j]^{-}$and rewriting the $j$-th, the $j+1$-th, and the $j+2$-th digits of the selected one, according to the rule shown in Table 2. $g^{\prime}$ denotes $\frac{1}{2} q_{j+1} r+x_{j+1} r+x_{j+2}$. The numbers in the rightmost column, e.g., $g^{\prime}+r^{2}$, mean theirs 2-digit nonredundant $r$-ary representations.

\section{Radix-2 version of the algorithm}

Different specific versions of the algorithm are possible, depending on the radix $r$, the redundancy factor $\rho$ of the correcting-digit set, the type of representation of the residual (carry-save or signed-digit), and the correcting-digit selection function. In this section, we show the details of a radix- 2 version of the algorithm.

We consider the case that the radix $r$ is 2 and the residual $W$ is represented in the carry-save form. When $r=2, \rho=1, a=1$, and the correcting-digit set is $\{-1,0,1\}$. Since $-4<W[j]<4, W[j]$ is represented by a two's complement carry-save form with 3 -bit integer part (including the sign bit).

The recurrences for radix 2 are

$$
P[j+1]:=P[j]+2^{-j-1} q_{j+1},
$$


Table 3. The rule for the extended on-the-fly conversion for $r=2$

\begin{tabular}{|c|c||c|c|c||c|c|c|}
\hline \multicolumn{2}{|c||}{} & \multicolumn{3}{c||}{$P[j+1]^{+}$} & \multicolumn{3}{c|}{$P[j+1]^{-}$} \\
\hline$g$ & $p[j]_{j}^{+}$ & $P[j]^{+} / P[j]^{-}$ & $p[j+1]_{j}^{+}$ & $p[j+1]_{j+1}^{+}$ & $P[j]^{+} / P[j]^{-}$ & $p[j+1]_{j}^{-}$ & $p[j+1]_{j+1}^{-}$ \\
\hline \hline \multirow{2}{*}{1,0} & 0 & $P[j]^{-}$ & 1 & $g+1$ & $P[j]^{-}$ & 0 & $g+1$ \\
\cline { 2 - 8 } & 1 & $P[j]^{+}$ & 0 & $g+1$ & $P[j]^{-}$ & 1 & $g+1$ \\
\hline \multirow{2}{*}{1,2} & 0 & $P[j]^{+}$ & 0 & $g-1$ & $P[j]^{-}$ & 1 & $g-1$ \\
\cline { 2 - 8 } & 1 & $P[j]^{+}$ & 1 & $g-1$ & $P[j]^{+}$ & 0 & $g-1$ \\
\hline
\end{tabular}

Table 4. The rule for generating the adder input for $r=2$

\begin{tabular}{|c|c||c|c|c|}
\hline$g^{\prime}$ & $p[j]_{j}^{+}$ & $P[j]^{+} / P[j]^{-}$ & $j$-th digit & $j+1$-th and $j+2$-th digits \\
\hline \hline-1 & & $P[j]^{-}$ & $p[j]_{j}^{-}$ & 3 \\
\hline \multirow{2}{*}{$0, \cdots, 3$} & 0 & $P[j]^{-}$ & 1 & $g^{\prime}$ \\
\cline { 2 - 5 } & 1 & $P[j]^{+}$ & 0 & $g^{\prime}$ \\
\hline \multirow{2}{*}{4} & & $P[j]^{+}$ & $p[j]_{j}^{+}$ & 0 \\
\hline \multicolumn{4}{|c|}{$g^{\prime}=q_{j+1}+2 x_{j+1}+x_{j+2}$}
\end{tabular}

and

$$
\begin{array}{r}
W[j+1]:=2 W[j]+Y y_{j+h+1} 2^{-h}+Z z_{j+h+1} 2^{-h} \\
-2 P[j] q_{j+1}-2^{-j-1} q_{j+1}^{2}
\end{array}
$$

Initially, $P[0]=X+2^{-1}$ and $W[0]=Y \cdot Y_{h}+Z$. $Z_{h}-X-2^{-2}$.

To obtain the correction-digit selection function, we first get the values of $L_{k}$ and $U_{k}$ from (9) and (10). $U_{-1}=-2^{-h+1}, L_{0}=-2 P[j]+2^{-j-1}, U_{0}=2 P[j]+$ $2^{-j-1}-2^{-h+1}$, and $L_{1}=0$. As stated in subsection 3.2 , a single selection function is possible for all $j$. Since $P[j] \geq 2^{-1}, \max \left(L_{0}\right)=-1$ and $\min \left(U_{0}\right)=1-2^{-h+1}$. $\min \left(U_{-1}\right)=-2^{-h+1}$ and $\max \left(L_{1}\right)=0$. Therefore, from (12) and (13), $m_{0} \geq-1, m_{0}+2^{-t}+2^{-h+1} \leq$ $-2^{-h+1}, m_{1} \geq 0$, and $m_{1}+2^{-t}+2^{-h+1} \leq 1-2^{-h+1}$ must be satisfied. We can choose $m_{0}=-1$ and $m_{1}=0$ by letting $t \geq 1$ and $h \geq 3$. Therefore, we let $m_{0}=-1$, $m_{1}=0, t=1$ and $h=3$.

In the radix- 2 version of the algorithm, we first set the initial values of the residual $W[0]$ and the pair representing the partial result $\left(P[0]^{+}, P[0]^{-}\right)$. Then, we perform $n$ iterations. In each iteration, we perform the following calculations.

1. Select the correcting-digit $q_{j+1}$ from $\{-1,0,1\}$, by examining the most significant 5 bits of the shifted residual $2 W[j]$. Recall that $W[j]$ has 3 -bit integer part and $t=1$.

2. Generate the partial products $Y y_{j+4}$ and $Z z_{j+4}$.

3. Generate

the adder input $-2 q_{j+1}\left(P[j]+2^{-j-2} q_{j+1}\right)$ from $q_{j+1}$ and $\left(P[j]^{+}, P[j]^{-}\right)$. The rule is shown in Table 4 . Note that $g^{\prime}=q_{j+1}+2 x_{j+1}+x_{j+2}$ and that the numbers in the rightmost column mean their 2-bit nonredundant binary representations.

4. Calculate the recurrence on the residual for obtaining $W[j+1]$ by means of carry-save additions.

5. Obtain the pair $\left(P[j+1]^{+}, P[j+1]^{-}\right)$representing the partial result from $q_{j+1}$ and $\left(P[j]^{+}, P[j]^{-}\right)$by means of the extended on-the-fly conversion. The rule is shown in Table 3.

Finally, we obtain $P$ from $\left(P[n]^{+}, P[n]^{-}\right) . P$ is either the one obtained by rewriting the least significant bit of $P[n]^{-}$to 1 or the one obtained by rewriting the least significant bit of $P[n]^{+}$to 0 , accordingly as the least significant bit of $P[n]^{+}$, i.e., $p[n]_{n}^{+}$is 0 or 1 .

The radix- 2 version of the algorithm is summarized as follows:

\section{Algorithm [3DEN_R2]}

Step 1:

$$
\begin{aligned}
& P[0]^{+}:=\left[10.0 x_{2} \cdots x_{n}\right] \\
& P[0]^{-}:=\left[00.0 x_{2} \cdots x_{n}\right] \\
& W[0]:=Y \cdot Y_{3}+Z \cdot Z_{3}-X-2^{-2}
\end{aligned}
$$

Step 2:

for $j:=0$ to $n-1$ do

begin

$$
q_{j+1}:=\left\{\begin{array}{rccc}
-1 & \text { if } & \hat{V}[j] & \leq-\frac{3}{2} \\
0 & \text { if } & -1 \leq & \hat{V}[j] \\
1 & \text { if } & \hat{V}[j] & \geq 0
\end{array} \leq-\frac{1}{2} ;\right.
$$

( $\hat{V}[j]$ : truncation of $2 W[j]$ to 1 fractional bit.) $W[j+1]:=2 W[j]+Y y_{j+4} 2^{-3}+Z z_{j+4} 2^{-3}$

$$
-2 q_{j+1}\left(P[j]+2^{-j-2} q_{j+1}\right)
$$


(Carry-save additions.)

$\left(P[j]+2^{-j-2} q_{j+1}\right.$ is generated by Table 4.$)$

Obtain $P[j+1]^{+}$and $P[j+1]^{-}$by Table 3 ; end;

Step 3:

Obtain $P$ from $P[n]^{+}$and $P[n]^{-}$;

\section{Implementations of the radix-2 ver- sion of the algorithm}

Any version of the algorithm can be implemented as a sequential circuit or a combinational circuit. Pipelining can also be used. Here, we consider implementations of the radix-2 version of the algorithm, i.e., Algorithm [3DEN_R2], discussed in the previous section.

First, we consider circuitry for performing one iteration of the 'for' loop in Step 2. It consists of the following circuits.

1. A circuit for examining the most significant 5 bits of $2 W[j]$ and selecting the correcting-digit $q_{j+1}$.

2. A circuit for generating partial products $Y y_{j+4}$ and $Z z_{j+4}$, which consists of AND gates.

3. A circuit for generating the adder input $-2 q_{j+1}\left(P[j]+2^{-j-2} q_{j+1}\right)$, which mainly consists of selectors.

4. A circuit for calculating the recurrence on the residual for obtaining $W[j+1]$, which mainly consists of carry-save adders.

5. A circuit for obtaining $\left(P[j+1]^{+}, P[j+1]^{-}\right)$, which mainly consists of selectors.

The delay of the circuit for one iteration is a small constant independent of $n$. Its amount of hardware is proportional to $n$. It has a regular linear array structure with a bit-slice feature.

Now we consider implementation of the algorithm as a combinational circuit. It is constructed by connecting a circuit for Step 1 which mainly consists of carry-save adders, $n$ copies of the circuit for one iteration of Step 2 discussed above, and a circuit for Step 3 which mainly consists of a selector, in series. The delay of the circuit is proportional to $n$. The amount of hardware is proportional to $n^{2}$. It has a regular 2-dimensional array structure suitable for VLSI implementation.

Next, we consider implementation of the algorithm as a sequential circuit which performs one iteration of Step 2 in each clock cycle. The circuit consists of a combinational circuit part and registers. The combinational circuit part consists of the circuits discussed above. We need registers REG-W, REG-PP, REG-PM,
REG-Y, REG-Z, for storing $W[j], P[j]^{+}, P[j]^{-}, Y$, and $Z$, respectively. Since $W[j]$ is in carry-save form, REGW has two flip-flops per place. Since we need $x_{j+1}$ and $x_{j+2}$ in the generation of $P[j+1]^{+}, P[j+1]^{-}$, and the adder input, we need a shift register SREG-X for storing $X$, whose content is shifted one position to the left in each cycle. We have to keep a copy of $p[j+1]_{j+1}^{+}$ separately from $P[j+1]^{+}$for these calculations in the next cycle. We also need two shift registers SREG-Y and SREG-Z for storing $Y$ and $Z$ besides REG-Y and REG-Z, because we need $y_{j+4}$ and $z_{j+4}$ for generating the partial products. Furthermore, we need a shift register SREG-J for indicating the $j$-th place, whose content is $2^{-j}$ and is shifted one position to the right in each cycle. Only one place of SREG-J keeps 1 and the others keep 0. Using SREG-J, we can know the bits which must be rewritten in the generation of $P[j+1]^{+}$, $P[j+1]^{-}$, and the adder input.

We can also perform Step 1 using this circuit. First, we initialize REG-PP to $\left[10.0 x_{2} \cdots x_{n}\right]\left(=X+2^{-1}+1\right)$, REG-PM to $\left[00.0 x_{2} \cdots x_{n}\right]\left(=X+2^{-1}-1\right)$, REG$\mathrm{W}$ to $\left[111.1110 \bar{x}_{2} \bar{x}_{3} \cdots \bar{x}_{n-1} \bar{x}_{n}\right]+[111.111110 \cdots 01]$ (= $\left(-X-2^{-2}\right) 2^{-3}$ in carry-save form) where $\bar{x}_{i}$ is the complement (logical inverse) of $x_{i}$, and SREG-J to $2^{3}$. Then, we execute 3 cycles (for $j=-3, j=-2$ and -1 ) with letting $q_{j+1}$ be 0 , without updating REG-PP nor REG-PM.

We can also perform Step 3 using the circuit. We execute one cycle (for $j=n$ ) with letting $q_{j+1}$ be 0 , then we can find $P$ in PEG-PP (down to $n$-th place).

Therefore, using the circuit, we can perform $n$-bit Euclidean norm computation in $n+4$ clock cycles. The length of the clock cycle is a small constant independent of $n$. The amount of hardware is proportional to $n$. It has a regular linear array structure with a bitslice feature. Figure 1 illustrates a block diagram of the circuit.

Of course, we can construct a sequential circuit which performs more than one iterations of Step 2 per clock cycle.

\section{Conclusion}

We have proposed a digit-recurrence algorithm for computing the Euclidean norm of a $3-\mathrm{D}$ vector which often appears in 3-D Computer Graphics. We have shown the general algorithm and discussed on the details of a radix- 2 version of the algorithm and its implementations. We have developed several techniques for extending the conventional digit-recurrence algorithms for division and square rooting. Among them, the technique for extending the one-the-fly conversion seems especially useful. Different specific versions of 


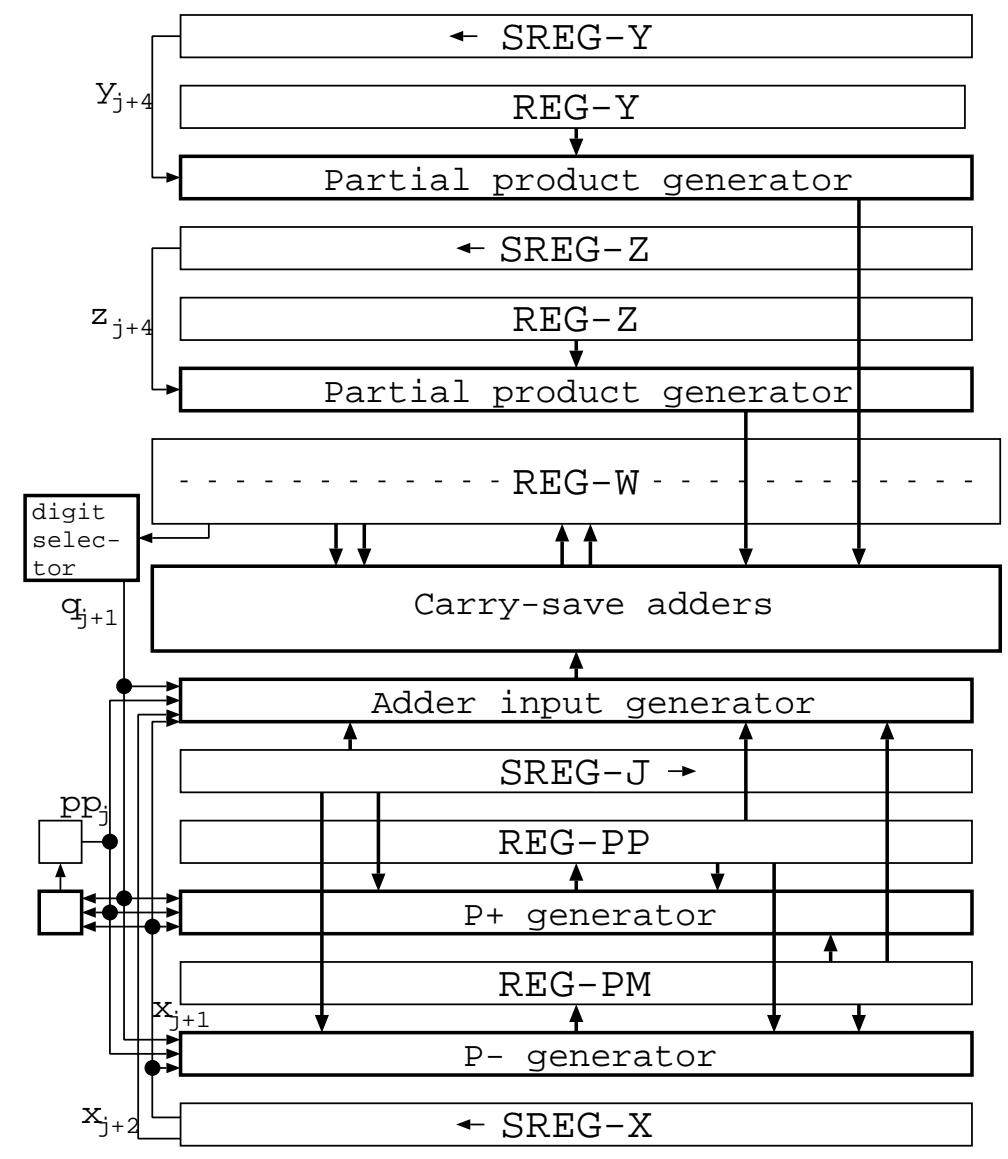

Figure 1. Block diagram of a sequential circuit based on [3DEN_R2]

the algorithm are possible, depending on the radix, the redundancy factor of the correcting-digit set, the type of representation of the residual, and the correctingdigit selection function.

Any version of the algorithm can be implemented as a sequential circuit or a combinational circuit. Pipelining can also be used. A sequential circuit performing one or several steps of the recurrence in one clock cycle has a regular linear array structure with bit-slice feature suitable for VLSI implementation. Its amount of hardware is proportional to $n$ where $n$ is the digit length of the vector components. The length of the clock cycle is a constant independent of $n$. The required number of clock cycles is proportional to $n$. A combinational circuit based on the algorithm has a regular 2-dimensional array structure suitable for VLSI implementation. Its delay is proportional to $n$ and its amount of hardware is proportional to $n^{2}$.

\section{References}

[1] A. Avizienis. Signed-digit number representations for fast parallel arithmetic, IRE Trans. Elec. Comput., EC10: 389-400, Sep. 1961.

[2] M. D. Ercegovac and T. Lang. On-the-fly conversion of redundant into conventional representations, IEEE Trans. Comput., C-36(7): 895-897, July 1987.

[3] M. D. Ercegovac and T. Lang. Division and Square Root - Digit-Recurrence Algorithms and Implementations, Kluwer Academic Publishers, 1994.

[4] M. D. Ercegovac and T. Lang. Radix-4 square root without initial PLA, Proc. 9th Symposium on Computer Arithmetic, 162-168, Sept. 1989. 